\title{
BASIC SETS OF INVARIANTS FOR FINITE REFLECTION GROUPS
}

\author{
BY LEOPOLD FLATTO ${ }^{1}$
}

Communicated by J. K. Moser, February 21, 1968

1. Introduction. Let $V$ be an $n$-dimensional vector space over a field $K$ of characteristic zero. Let $G$ be a finite group of linear transformations of $V . G$ acts naturally as a group of automorphisms of the ring of polynomials $K[x]$ if we define $(g P)(x)=P\left(g^{-1} x\right)$ for $g \in G$, $P(x) \in K[x]$. The polynomials which are invariant under $G$ form an algebra $I$ over $K$ called the algebra of invariants of $G$. A linear transformation is said to be a reflection if it has finite order and leaves fixed an (n-1)-dimensional hyperplane, called its reflecting hyperplane. $G$ is a finite reflection group if it is of finite order and is generated by reflections. Chevalley [5] has proved that for finite reflection groups, $I$ has an integrity basis consisting of $n$ algebraically independent forms $I_{1}, \cdots, I_{n}$. Furthermore, Shephard and Todd [10] have shown that this property of $I$ characterizes the finite reflection groups.

If $K$ is the real field $R$, then $G$ leaves invariant a positive definite quadratic form [2] so that $G$ is orthogonal after a linear change of variables. Coxeter [3], [4] has classified all irreducible finite orthogonal reflection groups and has computed the degrees $m_{1}, \cdots, m_{n}$ of the forms $I_{1}, I_{2}, \cdots, I_{n}$. These degrees are independent of the particularly chosen basis. We provide a method for computing an explicit integrity basis of $I$ for these groups. We will relate this problem to a certain mean value problem.

2. Construction of the basic set of invariants. We now state several theorems and sketch some of the proofs. Full details will appear elsewhere. Our first theorem yields a formula for the product of homogeneous invariants forming an integrity basis of $I$.

TheOREM 2.1. Let $G$ be an irreducible finite orthogonal reflection group acting on the real n-dimensional space $E_{n}$. Let $P_{m}(x, y)$ $=\sum_{\sigma \in G}(x \cdot \sigma y)^{m} \quad(1 \leqq m<\infty)$, where $x \cdot y=x_{1} y_{1}+\cdots+x_{n} y_{n}$. Let $J(x, y)=\partial\left(P_{m_{1}}, \cdots, P_{m_{n}}\right) / \partial\left(x_{1}, \cdots, x_{n}\right)$, where $m_{1}, \cdots, m_{n}$ denote the respective degrees of the basic invariant forms $I_{1}, \cdots, I_{n}$. Then $J(x, y)=\prod_{i=1}^{n} J_{i}(y) \prod_{i=1}^{r} L_{i}(x)$. The $J_{i}$ 's are homogeneous invariants

${ }^{1}$ Supported by National Science Foundation Grant GP-7352. 
(deg $J_{i}=m_{i}$ ) forming an integrity basis for $I$. The $L_{i}$ 's are linear forms and $L_{i}(x)=0(1 \leqq i \leqq r)$ are the $r$ reflecting hyperplanes corresponding to the reflections of $G$.

To prove the above theorem, we first establish the following lemma.

Lemma: Let $\odot$ be the ideal generated by $P_{m}(x, y)$, where $1 \leqq m<\infty$ and $y$ ranges over all vectors. Let $S_{0}$ be the ideal generated by all invariants of $G$ vanishing at 0 . Then $\rho=S_{0}$.

Proof. Let $f(x)$ be a continuous function on $E_{n}$ satisfying the mean value property

$$
f(x)=\frac{1}{|G|} \sum_{\sigma \in G} f(x+t \sigma y) \quad \text { for } x \in E_{n}, t>0,
$$

$y$ denoting a fixed vector $\neq 0$ and $|G|=$ order of $G$. The vectors $\sigma y(\sigma \in G)$ do not lie in a hyperplane, as $G$ is irreducible. It follows [9] that (2.1) is equivalent to

$$
f(x) \in C^{\infty} \quad \text { and } \quad P_{m}\left(\frac{\partial}{\partial x}, y\right) f=0, \quad 1<m<\infty .
$$

It is shown in [11] that the mean value property

$$
f(x) \in C \text { and } f(x)=\frac{1}{|G|} \sum_{\sigma \in G} f(x+\sigma y) \quad \text { for all } x \text { and } y
$$

is equivalent to

$$
f(x) \in C^{\infty} \text { and } s\left(\frac{\partial}{\partial x}\right) f=0, \quad s \in S_{0} .
$$

Now (2.3) is clearly equivalent to (2.1) holding for all $y$. We conclude that (2.4) is equivalent to (2.2) holding for all $y$. It follows that $S_{0}=\rho[6]$.

We now outline the proof of Theorem 2.1.

Proof of Theorem 2.1. Let $\tau \in G$. Then $P_{m}(\tau x, y)=\sum_{\sigma \in G}(\tau x \cdot \sigma y)^{m}$ $=\sum_{\sigma \in G}\left(x \cdot \tau^{-1} \sigma y\right)^{m}=\sum_{\sigma \in G}(x \cdot \sigma y)^{m}=P_{m}(x, y)$. Thus, for fixed $y$, $P_{m}(x, y)$ is an invariant of $G$ and is therefore a polynomial in $I_{1}(x)$, $\cdots, I_{n}(x)$. The $m_{i}$ 's are distinct [4], so that we may assume $m_{1}<m_{2}$ $<\cdots<m_{n}$. A standard degree argument then shows

$$
P_{m_{i}}(x, y)=Q_{i}(x, y)+J_{i}(y) I_{i}(x), \quad 1 \leqq i \leqq n,
$$

where $Q_{1}=0, Q_{i} \in\left(I_{1}(x), \cdots, I_{i-1}(x)\right)(2 \leqq i \leqq n), J_{i}(y)$ is a homogeneous polynomial of degree $m_{i}$. $\left(I_{1}(x), \cdots, I_{i-1}(x)\right)$ denotes as 
usual the ideal generated by $I_{1}(x), \cdots, I_{i-1}(x)$. A direct computation yields $\partial\left(P_{m_{1}}, \cdots, P_{m_{n}}\right) / \partial\left(x_{1}, \cdots, x_{n}\right)=J_{i}(y) \cdots J_{n}(y)$ $\partial\left(I_{1}, \cdots, I_{n}\right) / \partial\left(x_{1}, \cdots, x_{n}\right)$. It is shown in [4] that $\partial\left(I_{1}, \cdots, I_{n}\right) / \partial\left(x_{1}, \cdots, x_{n}\right)=\prod_{i=1}^{r} L_{i}(x)$. We will now show that $J_{i}(y)(1 \leqq i \leqq n)$ is an invariant of $G$ and $\partial\left(J_{1}, \cdots, J_{n}\right) / \partial\left(y_{1}, \cdots, y_{n}\right)$ $\neq 0$. This condition, which is equivalent to saying that $J_{1}, \cdots, J_{n}$ are algebraically independent, is precisely the criterion that the $J_{i}$ 's form and integrity basis of $I$ [10]. We first obtain a formula for the $J_{i}$ 's.

We employ the following notation. For any sequence of nonnegative integers $a_{1}, \cdots, a_{n}$ let $a=\left(a_{1}, \cdots, a_{n}\right), a !=a_{1} ! \cdots a_{n} !,|a|$ $=a_{1}+\cdots+a_{n}, x^{a}=x_{1}^{a_{1}} \cdots x_{n}^{a_{n}}$. Thus $(x \cdot y)^{m}=\sum_{|a| m m} m ! / a !\left(x^{a} y^{a}\right)$. Now

$$
\begin{aligned}
\sum_{\sigma_{1} \in G} \sum_{\sigma_{2} \in G}\left(\sigma_{1} x \cdot \sigma_{2} y\right)^{m} & =\sum_{\sigma_{1} \in G} \sum_{\sigma_{2} \in G}\left(x \cdot \sigma_{1}^{-1} \sigma_{2} y\right)^{m} \\
& =\sum_{\sigma_{1} \in G} \sum_{\sigma_{2} \in G}\left(x \cdot \sigma_{2} y\right)^{m}=|G| P_{m}(x, y) .
\end{aligned}
$$

Hence

$$
\begin{aligned}
P_{m}(x, y) & =\frac{1}{|G|} \sum_{\sigma_{1} \in G} \sum_{\sigma_{2} \in G} \sum_{|a|=m} \frac{m !}{a !}\left(\sigma_{1} x\right)^{a}\left(\sigma_{2} y\right)^{a} \\
& =\frac{1}{|G|} \sum_{|a|=m} \frac{m !}{a !} J_{a}(x) J_{a}(y),
\end{aligned}
$$

where $J_{a}(x)=\sum_{\sigma \in G}(\sigma x)^{a}$. Since $J_{a}(x)$ is invariant under $G$, we have for $m=m_{i}$

$$
J_{a}(x)=F_{a}\left(I_{1}(x), \cdots, I_{i-1}(x)\right)+c_{a} I_{i}(x), \quad|a|=m_{i},
$$

where $F_{a}$ is a polynomial in $I_{1}, \cdots, I_{i-1}\left(F_{a}=0\right.$ for $\left.|a|=m_{1}\right)$ and the $c_{a}$ 's are constants. As a polynomial in $x_{1}, \cdots, x_{n}, F_{a}$ is homogeneous of degree $m_{i}$. If $c_{a}=0$ for all $a\left(|a|=m_{i}\right)$, then we readily conclude that $I_{i} \notin \rho$, contradicting the lemma. Thus $c_{a} \neq 0$ for some $a$, $|a|=m_{i}$. Substituting (2.8) into (2.7) and comparing with (2.5) we obtain,

$$
\begin{aligned}
J_{i}(y)= & \frac{1}{|G|} \sum_{|a|=m_{i}} \frac{m !}{a !} c_{a} F_{a}\left(I_{1}(y), \cdots, I_{i-1}(y)\right) \\
& +\left(\frac{1}{|G|} \sum_{|a|=m i} \frac{m !}{a !} c_{a}^{2}\right) I_{i}(y),
\end{aligned}
$$

so that $J_{i}(y)$ is invariant under $G(1 \leqq i \leqq n)$. A direct computation shows 


$$
\frac{\partial\left(J_{1}, \cdots, J_{n}\right)}{\partial\left(y_{1}, \cdots, y_{n}\right)}=\frac{1}{|G|^{n}} \prod_{i=1}^{n}\left(\sum_{|a|=m_{i}} \frac{m !}{a !} c_{a}^{2}\right) \frac{\partial\left(I_{1}, \cdots, I_{n}\right)}{\partial\left(y_{1}, \cdots, y_{n}\right)} .
$$

Since $\quad \sum_{|a|=m_{i}}(m ! / a !) c_{a}^{2} \neq 0$ for $1 \leqq i \leqq n$, we conclude $\partial\left(J_{1}, \cdots, J_{n}\right) / \partial\left(y_{1}, \cdots, y_{n}\right) \neq 0$. We obtain immediately from Theorem 2.1 the

Corollary. Let $y$ be a fixed vector $\neq 0 . P_{m_{1}}(x, y), \cdots, P_{m_{n}}(x, y)$ form an integrity basis of $I$ iff $J_{1}(y) \cdots J_{n}(y) \neq 0$.

The following theorem gives an algorithm for computing the $J_{i}$ 's.

THEOREM 2.2. Let $J(x, y)=\left(\partial P_{i} / \partial x_{j}\right)$. Choose from the first $i$ rows of $J(x, y)$ an $i \times i$ minor $D_{i}(x, y)$ which is not identically zero. Then $D_{i}(x, y)=A_{i}(x) B_{i}(y)$, where $A_{i}(x)$ and $B_{i}(y)$ are polynomials not identically zero. $B_{i}(y)=J_{i}(y) \cdots J_{i}(y)$ so that $J_{i}(y)=B_{i}(y) / B_{i-1}(y)$ $(1 \leqq i \leqq n)$ with $B_{0}(y)=1$.

It is shown in [11] that the solution space to (2.4) is given by $D \Pi$ where $D \Pi$ denotes the linear span of the partial derivatives of $\Pi(x)$ $=\prod_{i=1}^{n} L_{i}(x)$. Let $\vartheta_{y}$ be the ideal generated by $P_{m}(x, y)(1 \leqq m<\infty)$ where $y$ is a fixed vector $\neq 0$. The solution space $S$ to (2.1) (or its equivalent (2.2)) $=D \Pi$ iff $S_{0}=\mathcal{P}_{y}$ [6]. It follows easily from (2.5) that $S_{0}=\mathcal{P}_{y}$ iff $J_{1}(y) \cdots J_{n}(y) \neq 0$. We thus obtain

THEOREM 3.1. Let $y$ be a fixed vector $\neq 0$. The solution space $S$ to $(2.1)$ $=D I I$ iff $J_{1}(y) \cdots J_{n}(y) \neq 0$.

3. The "vertex" conjecture. In view of Theorem (3.1) it is natural to ask whether there exists some canonical procedure for obtaining a vector $y$ such that $J_{1}(y) \cdots J_{n}(y) \neq 0$. The symmetry groups of the regular polyhedra centered at the origin form a subclass of the irreducible finite orthogonal reflection groups [3]. For these groups, it is conjectured that $y$ may be chosen as any vertex of the regular polyhedron. We refer to this conjecture as the "vertex" conjecture. It is equivalent to the following theorem.

THEOREM 3.1. Let $y_{1}, \cdots, y_{N}$ denote the vertices of the $n$-dimensional polyhedron $\pi_{n}$ centered at the origin. Let $f(x)$ be continuous in the $n$ dimensional region $R$ and let it satisfy the mean value property

$$
f(x)=\frac{1}{N} \sum_{i=1}^{N} f\left(x+t y_{i}\right), \quad x \in R, \quad 0<t<\epsilon_{x} .
$$

Then the solution space $S$ to $(3.1)=D \Pi$. 
The "vertex" conjecture has previously been established for special polyhedra [1], [8], [12]. We have verified it for all cases, with the exception of the $n$-dimensional cube. In the latter case, it is equivalent to the following

Unsolved Problem. Let $P_{2 k}(x)=\sum_{ \pm}\left( \pm x_{1} \pm x_{2} \pm \cdots \pm x_{n}\right)^{2 k}$. Are $P_{2}(x), P_{4}(x), \cdots, P_{2 n}(x)$ algebraically independent?

\section{REFERENCES}

1. E. F. Beckenbach and M. Reade, Regular solids and harmonic polynomials, Duke Math J. 12 (1945), 629-644.

2. W. Burnside, Theory of groups of finite order, 2nd ed., Cambridge Univ. Press, New York, 1911.

3. H. S. M. Coxeter, Regular polytopes, Methuen, London, 1948.

4. - The product of the generators of a finite group generated by reflections, Duke Math J 18 (1951), 765-782.

5. C. Chevalley, Invariants of finite groups generated by reflections, Amer. J. Math. 77 (1955), 778-782.

6. E. Fischer, Über algebraische Modulsysteme und lineare homogene partiel Differentialgleichungen mit konstaten Koeffizienten, J. Reine Angew. Math. 140 (1911), 48-81.

7. L. Flatto, Functions with a mean value property, J. Math. Mech. 10 (1961), 11-18.

8. - Functions with a mean value property. II, Amer. J. Math. 85 (1963), 248-270.

9. A. Friedman and W. Littman, Functions satisfying the mean value property, Trans. Amer. Math. Soc. 102 (1962), 167-180.

10. G. C. Shephard and J. A. Todd, Finite unitary reflection groups, Canad. J. Math. 6 (1954), 274-304.

11. R. Steinberg, Differential equations invariant under finite reflection groups, Trans. Amer. Math. Soc. 112 (1964), 392-400.

12. J. L. Walsh, $A$ mean value theorem for polynomials and harmonic polynomials, Bull. Amer. Math. Soc. 42 (1936), 923-926.

Belfer Graduate School of Science, Yeshiva University 